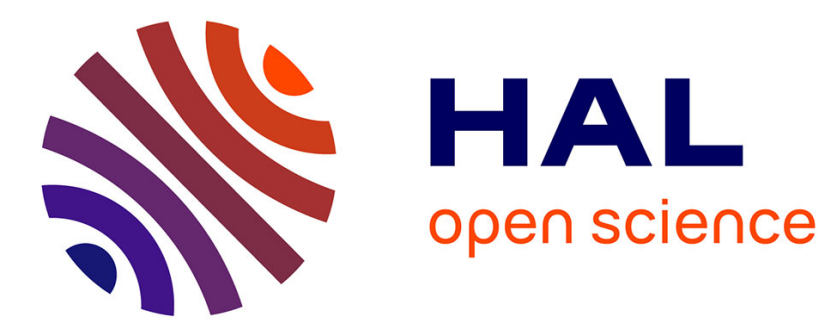

\title{
Scaling at low temperatures in the Ginzburg-Landau model
}

\author{
K. Uzelac, S. Barišić
}

\section{To cite this version:}

K. Uzelac, S. Barišić. Scaling at low temperatures in the Ginzburg-Landau model. Journal de Physique Lettres, 1977, 38 (2), pp.47-51. 10.1051/jphyslet:0197700380204700 . jpa-00231322

\section{HAL Id: jpa-00231322 https://hal.science/jpa-00231322}

Submitted on 1 Jan 1977

HAL is a multi-disciplinary open access archive for the deposit and dissemination of scientific research documents, whether they are published or not. The documents may come from teaching and research institutions in France or abroad, or from public or private research centers.
L'archive ouverte pluridisciplinaire HAL, est destinée au dépôt et à la diffusion de documents scientifiques de niveau recherche, publiés ou non, émanant des établissements d'enseignement et de recherche français ou étrangers, des laboratoires publics ou privés. 


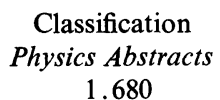

\title{
SCALING AT LOW TEMPERATURES IN THE GINZBURG-LANDAU MODEL
}

\author{
K. UZELAC and S. BARIŠIĆ $(*)$ \\ Institute of Physics of the University, Zagreb, Croatia, Yugoslavia \\ (Reçu le 15 janvier 1976, révisé le 27 octobre 1976, accepté le 7 décembre 1976)
}

\begin{abstract}
Résumé. - Nous examinons le modèle de Landau-Ginzburg, d'un paramètre d'ordre à $n$ composantes avec forces à courte portée, pour des dimensionnalités $d<2$. Pour $d$ et $n$ tels que $T_{\mathrm{c}}=0$, la solution de ce modèle se réduit à la solution du modèle de Heisenberg continu. Nous trouvons les exposants critiques pour la transition à $T_{\mathrm{c}}=0$ et en particulier $\eta=2-d$.
\end{abstract}

\begin{abstract}
The $n$-component Landau-Ginzburg model with short range forces is examined for dimensionalities $d$ less than two. For $d, n$ such that $T_{\mathrm{c}}=0$, its solution reduces to the solution of the continuous Heisenberg-like model. The critical exponents and corresponding scaling laws are found for the $T_{\mathrm{c}}=0$ transitions and in particular $\eta=2-d$.
\end{abstract}

Much of the current work on phase transitions [1-3] is based upon the $d$-dimensional Ginzburg-Landau (G-L) functional

$$
H[\varphi(x)]=a \varphi^{2}(x)+b \varphi^{4}(x)+c[\nabla \varphi(x)]^{2} .
$$

Here $\varphi(x)$ is the $n$-component classical order parameter. Usually one assumes $a=a^{\prime}\left(T-T_{\mathrm{MF}}\right)$ and $a^{\prime}, T_{\mathrm{MF}}, b$ and $c$, positive and finite. In fact, our derivation is valid under somewhat less restrictive requirements on $a, b, c$ which will be stated in the course of the work.

In general, the above functional has to be associated with a large momentum cut-off $Q$. This cut-off is required for $d \geqslant 2$, where it regularizes the ultraviolet singularities. The well known procedure which deals with the cut-off $Q$ in the region $2 \leqslant d \leqslant 4$ is the renormalization group $[2,4]$. However, as has been already realized for $d=1[1,3,5]$, for $d<2$ the ultraviolet singularities are absent, i.e. the cut-off can be omitted $(Q=\infty)$.

The infrared singularities we have to deal with (for $d<2$ ) make $\Gamma_{\mathrm{c}}$ vanish at $d<2, n=\infty$, without invalidating the G-L model itself [6]. The model also proved meaningful $[1,3,5]$ for $d=1$ and $n$ arbitrary [1], and the present paper interpolates between the $d=1, n>1[1,5]$ and the $n=\infty, d<2[6]$ results.

(*) And Laboratoire de Physique des Solides, Université ParisSud, 91405 Orsay, France.
In this work we assume at the outset that $T_{\mathrm{c}}=0$ $(d<2)$. This leads us to the use of the saddle point method with respect to the fluctuations in the amplitude of the order parameter. It follows that the amplitude fluctuations are quenched in the lowest order calculation. This determines the critical behaviour around $T_{\mathrm{c}}=0$, provided that the coefficients of the so obtained $T$-dependences are consistent with the stability requirement for the system. We associate the zero of these coefficients with the borderline between $T_{\mathrm{c}}=0$ and $T_{\mathrm{c}} \neq 0$ regions for $d<2$ : on the borderline the lowest order saddle point approximation starts to break down and the coupling between phase and amplitude fluctuations becomes relevant.

This purely thermodynamical argument suggests a connection to the recent proof [7] that the singularities in the order parameter, related to the amplitude fluctuations, are topologically unstable to the right of the $d=n$ line of figure 1 . One is then tempted to think that the thermodynamical borderline coincides with the topological one. We know $[1,7]$ that actually they do coincide at $d=n=1$. At this point the point singularities to determine [8] the critical $[1,5]$ behaviour; for $n>1$ the amplitude fluctuations are unimportant [1] in the critical $[1,5]$ thermodynamics.

The results of this letter are thus the following : we determine the critical indices in the interior of the $T_{\mathrm{c}}=0$ region. Beside coinciding with the exact $T_{\mathrm{c}}=0$ results $[1,5]$, as already mentioned, these results are consistent with the results [4] for $d \gtrsim 2$, 


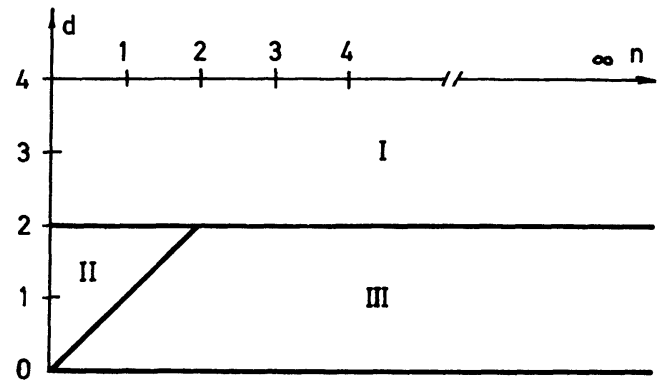

FIG. 1. - Three regions in the $n, d$ plane : region I with $Q$ finite, $T_{\mathrm{c}} \neq 0$, region II with $Q=\infty, T_{\mathrm{c}} \neq 0$, region III with $Q=\infty$, $T_{\mathrm{c}}=0$. The line between regions II and III is not yet well established. $n>2$ (cf. Fig. 1). It is further clear from our approach where the proof of the coincidence of the thermodynamical and topological borderline is to be searched for, but this proof is not obtained here.

Our present derivation is based on the homogeneity argument which is an extension to $0<d<2$ of our previous $d=1$ approach [5]. In fact we show here that the transfer matrix formulation $[1,3,5]$, natural in $d=1$, is a non essential step in the derivation [5] of the critical behaviour. We start therefore from the functional-integral definition of the correlation function $\Gamma$ and free energy $F$, which we present as functions of the parameters in the G-L functional.

$$
\begin{gathered}
\Gamma=\frac{\int D \varphi(x) \varphi\left(x_{1}\right) \varphi(0) \exp \left(-\frac{1}{T} \int_{x} H[\varphi(x)]\right)}{\int D \varphi(x) \exp \left(-\frac{1}{T} \int_{x} H[\varphi(x)]\right)}=\Gamma\left(X_{1}, \frac{a}{T}, \frac{b}{T}, \frac{c}{T}\right) \\
\exp \left(-\frac{L^{d} F}{T}\right)=\int D \varphi(x) \exp \left(-\frac{1}{T} \int_{x} H[\varphi(x)]\right)=\exp \left(-L^{d} \mathfrak{F}\left(\frac{a}{T}, \frac{b}{T}, \frac{c}{T}\right)\right)
\end{gathered}
$$

where $\int_{x} \equiv \int_{L^{d}} d^{d} x$, and $L^{d}$ is the volume.

Choosing the new variable $x^{\prime}=x / s$ and redefining the field variable by $\varphi\left(x^{\prime} . s\right)=\alpha \psi\left(x^{\prime}\right)$, we obtain from eqs. (2) and (3) the homogeneity relations

$$
\begin{gathered}
\Gamma\left(x_{1}, \frac{a}{T}, \frac{b}{T}, \frac{c}{T}\right)_{Q}=\alpha \Gamma\left(\frac{x_{1}}{s}, \alpha s^{d} \frac{a}{T}, \alpha^{2} s^{d} \frac{b}{T}, \alpha s^{d-2} \frac{c}{T}\right)_{Q . s} \\
\mathcal{F}\left(\frac{a}{T}, \frac{b}{T}, \frac{c}{T}\right)_{Q}=s^{-d} \mathcal{F}\left(\alpha s^{d} \frac{a}{T}, \alpha^{2} s^{d} \frac{b}{T}, \alpha s^{d-2} \frac{c}{T}\right)_{Q . s}
\end{gathered}
$$

which are valid for all $\alpha$ and $s$. Eqs. (4) and (5) are derived without specifying $d$ and $n$. However, they are not useful except when they connect systems with the same cut-offs and when the critical temperature dependence is explicitly exhibited. This is realized only in region III of figure 1 where $Q=\infty$ and $T_{\mathrm{c}}=0$.

In the following we shall thus restrict ourselves to this region. Before proceeding to the calculation of the critical behaviour it is convenient to express eqs. (4) and (5) in terms of dimensionless variables. This can be done by an appropriate choice of $\alpha$ and $s$ in (4) and (5). In this way we obtain

$$
\Gamma \equiv \Gamma\left(x_{1}, \frac{a}{T}, \frac{b}{T}, \frac{c}{T}\right)=-\frac{a}{b} \Gamma\left(\frac{x}{\xi_{0}},-\frac{T_{b}}{T}, \frac{T_{b}}{T}, \frac{T_{b}}{T}\right)
$$

and

$$
\mathfrak{F} \equiv \mathfrak{F}\left(\frac{a}{T}, \frac{b}{T}, \frac{c}{T}\right)=\xi_{0}^{-d} \mathfrak{F}\left(-\frac{T_{b}}{T}, \frac{T_{b}}{T}, \frac{T_{b}}{T}\right),
$$

where

$$
\xi_{0}=\sqrt{-\frac{c}{a}} \text { and } T_{b}=\left(-\frac{c}{a}\right)^{d / 2} \frac{a^{2}}{b} .
$$

Of course, the right hand sides of eqs. (6) and (7) also satisfy the homogeneity properties (4) and (5). We notice that parameters appear here only in the combinations, $a / b, c / a$ and $T_{b} / T$. The only assumptions we need for our next step is that $a<0, b>0, c>0$ and that, in the limit of small $T, T_{b} / T$ tends to infinity. It is apparent here that the displacive phase transition [9] $\left(a=0\right.$, i.e. $\left.T_{b}=0\right)$ is not covered by our discussion and represents a singular case in the $a, b, c$, parameter space. 
The next step consists in reducing our homogeneity relations to a form from which the critical exponents can be obtained.

Let us start by considering the correlation function. Applying the property (4) to eq. (6) by the substitution $\alpha=1$,

$$
s=\left(\frac{T_{b}}{T}\right)^{\frac{1}{2-d}}
$$

we get :

$$
\Gamma=\frac{-a}{b} \Gamma\left(\frac{x_{1}}{\xi_{0}}\left(\frac{T}{T_{b}}\right)^{\frac{1}{2-d}},-\left(\frac{T_{b}}{T}\right)^{\frac{2}{2-d}},\left(\frac{T_{b}}{T}\right)^{\frac{2}{2-d}}, 1\right) .
$$

It is now convenient to express the $n$-component vector $\varphi(x)$ as a product of its amplitude $|\varphi(x)|$ and the unit vector $\theta(x)$

$$
\varphi(x)=|\varphi(x)| \theta(x)
$$

The right hand side expression of eq. (9) then reads explicitly

$$
\begin{aligned}
& \Gamma\left(\tilde{x}_{1},-\left(\frac{T_{b}}{T}\right)^{\frac{2}{2-d}},\left(\frac{T_{b}}{T}\right)^{\frac{2}{2-d}}, 1\right)= \\
& \int D|\varphi(\tilde{x})| D \Omega(\tilde{x}) \exp \left[-\left(\frac{T_{b}}{T}\right)^{\frac{2}{2-d}} \int_{\tilde{x}}\left[-|\varphi(\tilde{x})|^{2}+|\varphi(\tilde{x})|^{4}\right]\right] \times \\
& =\frac{\int D\left|\exp \left[-\int_{\tilde{x}}|\nabla| \varphi(\tilde{x})|\theta(\tilde{x})|^{2}\right]\right| \varphi\left(\tilde{x}_{1}\right)|| \varphi(0) \mid \theta\left(\tilde{x}_{1}\right) \theta(0)}{\int D(\tilde{x}) \mid D \Omega(\tilde{x}) \exp \left[-\left(\frac{T_{b}}{T}\right)^{\frac{2}{2-d}} \int_{\tilde{x}}\left[-|\varphi(\tilde{x})|^{2}+|\varphi(\tilde{x})|^{4}\right]\right] \exp \left[-\int_{\tilde{x}}|\nabla| \varphi(\tilde{x})|\theta(\tilde{x})|^{2}\right]}
\end{aligned}
$$

where

$$
\tilde{x}=\frac{x}{\xi_{0}}\left(\frac{T}{T_{b}}\right)^{\frac{1}{2-d}}
$$

Since we assumed that near $T_{\mathrm{c}}=0 \quad \frac{T_{b}}{T} \gg 1$, we can apply the saddle point method to the amplitude integration. From the condition for a minimum

$$
\frac{\mathrm{d}}{\mathrm{d}|\varphi(\tilde{x})|}\left[-|\varphi(\tilde{x})|^{2}+|\varphi(\tilde{x})|^{4}\right]=0,
$$

it follows that $|\varphi(\tilde{x})|_{\min }=\frac{1}{\sqrt{2}}$ for all $\tilde{x}$.

By taking only the zeroth order term on the saddle point expansion we disregard completely the amplitude fluctuations which are strongly damped by the factor $\left(\frac{T_{b}}{T}\right)^{\frac{2}{2-d}} \gg 1$. In this way we find that $\Gamma$ of eq. (11) is equal to

$$
\frac{1}{2} \frac{\int D \Omega(\tilde{x}) \theta\left(\tilde{x}_{1}\right) \theta(0) \exp \left(-\frac{1}{2} \int_{\tilde{x}}|\nabla \theta(\tilde{x})|^{2}\right)}{\int D \Omega(\tilde{x}) \exp \left(-\frac{1}{2} \int_{\tilde{x}}|\nabla \theta(\tilde{x})|^{2}\right)} .
$$


The important simplification comes from the fact that it is not necessary to carry out the functional integration (13). It is sufficient to notice that this integral is essentially equivalent to the correlation function of the continuous $0<d<2$ Heisenberg like model for a special value of the coupling constant. It defines therefore a finite function $f\left(\tilde{x}_{1}\right)$, so that the full correlation function has then the form

$$
\Gamma=\frac{-a}{2 b} f\left(\tilde{x}_{1}\right)=\frac{-a}{2 b} f\left(\frac{x}{\xi_{0}}\left(\frac{T}{T_{b}}\right)^{\frac{1}{2-d}}\right) .
$$

The function $f$ is based on the gradient term $\nabla \boldsymbol{\theta}$ and does not exist for $d=0$. We can now conclude that the $T_{\mathrm{c}}=0$ critical behaviour of the homogeneous function

$$
\Gamma \simeq \frac{1}{x^{(\eta+d-2)}} f(x / \xi)
$$

is described by the correlation length

$$
\xi=c_{2} \xi_{0}\left(\frac{T_{b}}{T}\right)^{\frac{1}{2-d}}
$$

where $c_{2}$ is the coefficient discussed below, and by $\eta=2-d$. The assumption that $a, b$ and $c$ are constants in the limit $T=0$, gives then $v=\frac{1}{2-d}$, in agreement with previous $d=1[1,5]$ and $n=\infty[6]$ results.

The result $2-d-\eta=0$ can be understood more heuristically. While in general the function $\Gamma(x)$ is defined by

$$
\frac{1}{x^{(\eta+d-2)}} f(x / \xi)
$$

for $x \geqslant 1 / Q$, for $d<2$ (where $Q=\infty$ ) the validity of this expression extends to $x \rightarrow 0$, where it should coincide with the value of the autocorrelation function $\left\langle\varphi^{2}\right\rangle$. Since for $T_{\mathrm{c}}=0$ the problem reduces to that of the continuous Heisenberg-like system with fixed length of the order paraemeter-finite $\left\langle\varphi^{2}\right\rangle$, this means $2-d-\eta=0$.

The procedure of eqs. (9)-(14) can be also applied to the free energy $F$. Taking only the first terms in the saddle point expansion we obtain

$$
F=-\frac{a^{2}}{4 b}+c_{0} \frac{T}{T_{b}}+c_{1}\left(\frac{T}{T_{b}}\right)^{\frac{2}{2-d}} \cdot \frac{a^{2}}{4 b},
$$

where $c_{0}$ and $c_{1}$ are the appropriate coefficients. To this order the amplitude and phase integrations are separated. The first two terms come from the amplitude integration, while the third is of the same nature as the one retained in the correlation function and represents the singular part of the free-energy, $F_{\text {sing }}$. The coefficients $c_{2}$ and $c_{1}$ in eqs. (15) and (16) respec- tively are functions of $n$ and $d$ only. This is obvious from eqs. (13) and (14). Eq. (13) treated by the transfer matrix method at $d=1$ gives $c_{1,2} \sim(n-1)$. As already explained, we expect that more generally i.e. for $n>d$

$$
c_{1,2} \sim(n-d) .
$$

The proof of this conjecture would require the full solution of the weak coupling, continuous problem (eq. (13)).

If we introduce a symmetry breaking field $h$ as a small perturbation, it will affect in the first approximation only $F_{\text {sing }}$ giving

$$
F_{\text {sing }}=\frac{a^{2}}{b}\left(\frac{T}{T_{b}}\right)^{\frac{2}{2-d}} g\left[h \sqrt{\frac{-b}{a^{3}}}\left(\frac{T_{b}}{T}\right)^{\frac{2}{2-d}}\right] .
$$

Here $g$ is an unknown function, which becomes a constant when $h \rightarrow 0$. The critical exponents and the scaling laws follow from eq. (18). If, again, we assume that $a, b, c$, are constant in the limit $T \rightarrow 0$, we obtain

$$
\begin{aligned}
\Delta & =\frac{2}{2-d} \\
\gamma & =\alpha_{G}+2 \Delta-2=\frac{2}{2-d} \\
2-\alpha_{G} & =2 v \\
\alpha_{G} & =\alpha+1=\frac{2-2 d}{2-d} .
\end{aligned}
$$

One can see that some of the scaling laws are modified. This is due to the additional $k_{\mathrm{B}} T$ factor which enters in the definition of some critical exponents when $T_{\mathrm{c}}=0$. E.g. for

$$
C_{\mathrm{v}}=T \frac{\partial^{2} F}{\partial T^{2}} \sim \frac{1}{T^{\alpha}}
$$

we have $\alpha=\alpha_{G}-1$. Similarily, the susceptibility exponent $\gamma$ differs by one from the correlation function exponent.

Another feature is that the critical exponents are independent of $n$ which gives additional scaling laws, involving only two parameters, e.g.

$$
\eta=\frac{1}{v}
$$

The above homogeneity analysis can be also applied to the lattice anisotropy cross-over $d \rightarrow d^{\prime}$ where in the lower dimension $d^{\prime} T_{\mathrm{c}}=0$, while the upper dimensionality $d$ is associated with a finite $T_{\mathrm{c}}[5,6$, $10,11]$. Repeating the procedure of ref. [5] we find that the cross-over exponent $\varphi$ and the transition temperature shift exponent $\psi$ satisfy the relation

$$
\varphi=\psi=\gamma_{d^{\prime}}=\frac{2}{2-d^{\prime}} .
$$




\section{References}

[1] Balian, R. and Toulouse, G., Ann. Phys. 83 (1974) 28.

[2] Wilson, K. G. and Kogut, J., Phys. Rep. $12 C$ (1974) 75.

[3] Scalapino, D. J., Sears, M., Ferrel, R. A., Phys. Rev. $\mathrm{B} 6$ (1972) 3409.

[4] Migdal, A. A., Zh. E.T.F. 69 (1975) 1457; Brézin, E. and Zinn Justin, J., Phys. Rev. Lett. 36 (1976) 691.

[5] Barišić, S. and Uzelac, K., J. Physique 36 (1975) 1267.

[6] Barišıć, S. and Uzelac, K., J. Physique 36 (1975) 325. BARIŠIĆ, S., Fizika 8 (1976) 181.
[7] Toulouse, G., Bull. Soc. Fr. Phys. 24 (1976) 19.

[8] Krumhansl, J. A., SChrieffer, J. R., Phys. Rev. B 11 (1975) 3535.

[9] BeCK, H., SChNeIDeR, T. and Stoll, E., Phys. Rev. B 12 (1975) 5198.

[10] Scalapino, D. Y., Imry, Y., Pincus, P., Phys. Rev. B 11 (1975) 2042

[11] DieTerich, W., Z. Phys. 270 (1974) 239. 